\title{
Proteomic analysis revealed alterations of the Plasmodium falciparum metabolism following salicylhydroxamic acid exposure
}

This article was published in the following Dove Press journal:

Research and Reports in Tropical Medicine

7 September 201 I

Number of times this article has been viewed

\section{Marylin Torrentino- \\ Madamet' \\ Lionel Almeras ${ }^{2}$ \\ Christelle Travaillél \\ Véronique Sinou' \\ Matthieu Pophillat ${ }^{3}$ \\ Maya Belghazi ${ }^{4}$ \\ Patrick Fourquet ${ }^{3}$ \\ Yves Jammes ${ }^{5}$ \\ Daniel Parzy'}

'UMR-MD3, Université de la Méditerranée, Antenne IRBA de Marseille (IMTSSA, Le Pharo),

${ }^{2}$ Unité de Recherche en Biologie et Epidémiologie Parasitaires, Antenne IRBA de Marseille (IMTSSA, Le Pharo),

${ }^{3}$ Centre d'Immunologie de Marseille Luminy, Institut National de la Santé et de la Recherche Médicale, Centre National de la Recherche Scientifique, Université de la Méditerranée,

${ }^{4}$ Centre d'Analyse Protéomique de Marseille, Institut Fédératif de Recherche Jean Roche, Faculté de Médecine Nord, ${ }^{5}$ UMR-MD2, Physiologie et Physiopathologie en Conditions d'Oxygénations Extrêmes, Institut Fédératif de Recherche Jean Roche, Faculté de Médecine Nord, Marseille, France
Correspondence: Marylin Torrentino-Madamet UMR-MD3, Université de la Méditerranée, Antenne IRBA de Marseille (IMTSSA, Le Pharo), Allée du Médecin colonel Eugène Jamot - BP 60109,

I 3262 Marseille cedex 07, France

$\mathrm{Tel}+3349 \mid \quad 150$ । 14

Fax +33 49I I50 I64

Email madametm@imtssa.fr
Objectives: Although human respiratory metabolism is characterized by the mitochondrial electron transport chain, some organisms present a "branched respiratory chain." This branched pathway includes both a classical and an alternative respiratory chain. The latter involves an alternative oxidase. Though the Plasmodium falciparum alternative oxidase is not yet identified, a specific inhibitor of this enzyme, salicylhydroxamic acid (SHAM), showed a drug effect on P. falciparum respiratory function using oxygen consumption measurements. The present study aimed to highlight the metabolic pathways that are affected in $P$. falciparum following SHAM exposure.

Design: A proteomic approach was used to analyze the $P$. falciparum proteome and determine the metabolic pathways altered following SHAM treatment. To evaluate the SHAM effect on parasite growth, the phenotypic alterations of $P$. falciparum after SHAM or/and hyperoxia exposure were observed.

Results: After SHAM exposure, 26 proteins were significantly deregulated using a fluorescent two dimensional-differential gel electrophoresis. Among these deregulated proteins, some were particularly involved in energetic metabolism. And the combinatory effect of SHAM/hyperoxia seems deleterious for the growth of $P$. falciparum.

Conclusion: Our results indicated that SHAM appears to activate glycolysis and decrease stress defense systems. These data provide a better understanding of parasite biology.

Keywords: Plasmodium falciparum, salicylhydroxamic acid, hyperoxia, glycolysis, proteomic

\section{Background}

The malaria parasite Plasmodium falciparum possesses a mitochondrion with specific structural and physiological features. ${ }^{1-4}$ Unlike most other eukaryotes, malarial mitochondrial electron transport chain (mETC) activity appears to be primarily involved in de novo pyrimidine biosynthesis through the activity of dihydroorotate dehydrogenase rather than through an adenosine triphosphate (ATP) source. ${ }^{4-6}$ Intraerythrocytic stages of malaria parasites have been considered for a long time to rely primarily on anaerobic glycolysis for ATP production. However, recently five subunits of the $\mathrm{F}_{1}$ subcomplex and four $\mathrm{F}_{0}$ subunits of the mitochondrial $\mathrm{F}_{0} \mathrm{~F}_{1}$ ATP synthase have been reported in the $P$. falciparum genome, supporting the fact that oxidative phosphorylation could be functional. ${ }^{7}$ As parasite respiratory metabolism is distinct from human respiration, diverse inhibitors of the major complexes of the mETC have been used in chemotherapy ${ }^{8}$ and validated the importance of the mitochondrion in the growth of the asexual intraerythrocytic stage of $P$. falciparum. ${ }^{9,10}$ Moreover, 
the metabolic changes of the mitochondrion contribute to environmental adaptations of parasites during its life cycle. ${ }^{11}$ Effectively, in vivo, $P$. falciparum life cycle involves two hosts: the mosquito and humans during which the parasite is subjected to varying oxygen levels (from 5\% oxygen in human venous blood to $13 \%$ oxygen in the human lungs and $21 \%$ oxygen in mosquito salivary glands).

Study of the rotenone drug effect underlined the divergence of the first component (complex I) of the $P$. falciparum mETC. ${ }^{12-14}$ The eukaryotic complex I is classically composed of a rotenone-sensitive nicotinamide adenine dinucleotide hydride (NADH) dehydrogenase. In contrast, $P$. falciparum encodes a rotenone-insensitive alternative complex I (type II NADH dehydrogenase, PfNDH2). This complex I is comparable to type II NADH dehydrogenase found in plants. Not surprisingly, parasite mitochondria have an endosymbiotic origin probably resulting from algal symbiont into a protoeukaryote. ${ }^{15-17}$ Moreover, other mitochondrial drugs such as atovaquone inhibit electron transfer at the level of the bc1 complex (complex III) by interfering with the ubiquinol oxidation site of cytochrome $b$. The drug effect is lethal for the parasite, presumably by interruption of essential links to de novo pyrimidine biosynthesis and to the collapse of the mitochondrial transmembrane potential. ${ }^{18,19}$ This drug is already currently used in endemic regions in combination with proguanil (Malarone ${ }^{\circledR}$ ). ${ }^{20}$ Also, the combination of atovaquone and salicylhydroxamic acid (SHAM), a specific inhibitor of alternative oxidase (AOX), inhibits parasite growth synergistically. ${ }^{21,22}$ The AOX, which is absent from mammals, has been most extensively studied in higher plants. The plant respiratory pathway includes an alternative respiratory chain, cyanide-resistant, and the classical mETC, cyanidesensitive. The AOX does not appear to contribute directly to mitochondrial transmembrane potential or the energy balance of the cell. ${ }^{23,24}$ However, it can contribute indirectly by accepting electrons from enzymes that provide electrons to coenzyme $\mathrm{Q}$ for the preservation of tricarboxylic acid cycle (TCA) turnover and the balance of carbon metabolism and electron transport. From the mitochondrial transmembrane potential and oxygen consumption measurements in presence of $P$. falciparum mETC inhibitors, the evidence of an alternative respiratory pathway in which electrons are transferred directly from coenzyme Q to oxygen was underlined. ${ }^{25}$ This cyanide-resistant alternative respiratory pathway was inhibited by two inhibitors, propyl gallate and SHAM, which are specific inhibitors of AOX activity in other organisms. ${ }^{26-28}$ The AOX gene has been characterized in several organisms such as plants, yeast, bacterium, and notably from other parasites, Trypanosoma brucei and Cryptosporidium parvum. ${ }^{28-30}$ Nevertheless, until now, no gene encoding AOX has been detected in the $P$. falciparum genome but $60 \%$ of the predicted genes could not be assigned to orthologous functions.

In the present work, a two dimensional-differential gel electrophoresis (2D-DIGE) approach, combined with mass spectrometry (MS) analysis, was used to define the consequences of SHAM on the $P$. falciparum proteome. Studies were previously reported that the hyperoxia induces a cycle delay on $P$. falciparum of 4 hours with a biological adaptation of the parasite to next cycle. ${ }^{11,31}$ The deleterious effect of this drug on the in vitro parasite growth in hyperoxia, the involved metabolic pathways, and its mode of action on glycolysis are discussed, supporting the hypothesis that the energetic metabolism may indeed provide an attractive chemotherapeutic target.

\section{Methods SHAM or/and hyperoxia exposure of $P$. falciparum cultures}

The in vitro antimalarial activity of SHAM was determined using the isotopic semi microtest method as previously described by Desjardins et al. ${ }^{32}$ Drug concentrations were from 0.5 to $600 \mu \mathrm{M}$. The $\mathrm{IC}_{50}$, ie, the drug concentration corresponding to $50 \%$ of the uptake of $\left[{ }^{3} \mathrm{H}\right]$ hypoxanthine by the parasite in drug-free control wells, was determined by nonlinear regression analysis of log dose-response curves.

The 3D7 $P$. falciparum strain was cultured in human A+ erythrocytes, as previously described. ${ }^{33}$ Cultures were tightly synchronized (with 4 hour intervals) using combined D-sorbitol and CS columns on a VarioMACS (Miltenyi Biotec, Paris, France) according to standard procedures. ${ }^{34,35}$

To evaluate the effect of SHAM, hyperoxia, and the combinatory effect, cultures were adapted to hyperoxic conditions ( $21 \%$ oxygen, $5 \%$ carbon dioxide, $74 \%$ nitrogen gas mixture) and regularly synchronized during six $P$. falciparum parasites' cycles before the addition of SHAM. The effects of SHAM alone and combined SHAM/hyperoxia were observed at $0,24,32,48$, and 78 hours ( 0 hours corresponding to SHAM addition at the ring stage). Viability, parasitemia, and morphology were monitored daily by examining blood smears stained with RAL 555 (RAL Diagnostics, Martillac, France) from SHAM addition at the ring stage.

To test the SHAM effects on $P$. falciparum proteome ( $3 \%$ parasitemia and $6 \%$ hematocrit), the drug was added to $25 \mathrm{~mL}$ culture medium at $\mathrm{IC}_{50}$ and the parasites were further 
incubated for 12 hours and harvested. For each proteomic experiment, four biological replicates were performed.

\section{Protein extraction}

After SHAM pressure, parasitized erythrocytes (late ring stages aged 16-20 hours) were washed three times in phosphate buffered saline (PBS) medium (Invitrogen, Cergy Pontoise, France) and lysed in cold water-saponin $(0.1 \%$, Sigma, St Louis, MO) for 10 minutes. The lysate was then centrifuged at $1500 \mathrm{~g}$ for 5 minutes. The supernatant was discarded and the pellet containing free parasites was recovered by washing in cold PBS medium followed by a centrifugation step ( $1500 \mathrm{~g}$ for 5 minutes). The free parasites were washed until the supernatant became colorless. The pellet was then suspended in 4\% (w/v) CHAPS (Sigma) and disrupted by ultrasonication (Vibracell 72412; Bioblock Scientific, Illkirch, France) 60 seconds on ice at maximum amplitude. The lysate was then centrifuged at $16,100 \mathrm{~g}$ for 15 minutes. The supernatant was further precipitated with $100 \%$ acetone (Sigma). The protein concentration for each sample was estimated using the BioRad Lowry-based DC assay (BioRad Laboratories, Hercules, CA), according to the manufacturer's instructions. Total proteins were suspended in standard cell lysis buffer (7 M urea, $2 \mathrm{M}$ thiourea, 4\% CHAPS, $30 \mathrm{mM}$ Tris base, $\mathrm{pH} 8.5$ [Sigma]) to obtain a protein concentration adjusted to $2.5 \mu \mathrm{g} / \mu \mathrm{L}$. Protein samples were minimally labeled with CyDye according to the manufacturer's protocols (GE Healthcare, Piscataway, NJ). ${ }^{36}$

Briefly, protein extracts $(50 \mu \mathrm{g})$ were labeled with 400 pmol of CyDye, freshly dissolved in anhydrous dimethyl formamide (Sigma) and incubated on ice for 30 minutes in the dark. The reaction was quenched with $1 \mu \mathrm{L}$ of free lysine (10 nM, Sigma) by incubating for 10 minutes on ice. An equal volume of $2 \times$ sample buffer ( $8 \mathrm{M}$ urea, $2 \mathrm{M}$ thiourea, $4 \%(\mathrm{w} / \mathrm{v})$ CHAPS, $10 \mathrm{mM}$ Dithiothreitol (DTT) and 1\% (v/v) IPG buffer 3-10 [GE Healthcare]) was added to the CyDye-labeled sample. The mixture of labeled proteins was then separated by 2D-DIGE.

\section{D electrophoresis of parasite proteins, image analysis, and in-gel digestion}

Isoelectric focusing was performed on $18 \mathrm{~cm} \mathrm{pH} \mathrm{3-10} \mathrm{linear}$ IPG strips (GE Healthcare). Destreak buffer containing 1\% (v/v) IPG buffer $\mathrm{pH} 3-10$ was used for overnight rehydration of IPG strips. The samples were applied at the acidic end of the IPG strip using a cup-loading technique. Isoelectric focusing was carried out on an Ettan IPGphor II (GE Healthcare) electrophoresis unit at $20^{\circ} \mathrm{C}$ for a total of $45 \mathrm{kVh}$ (ramp to $300 \mathrm{~V}$ in 3 hours, ramp to $1000 \mathrm{~V}$ in 6 hours, ramp to $8000 \mathrm{~V}$ in 3 hours, hold at $8000 \mathrm{~V}$ for 4 hours). IPG strips were equilibrated in a equilibration buffer containing $50 \mathrm{mM}$ Tris hydrochloride, $\mathrm{pH} 8.6,6 \mathrm{M}$ urea, 2\% sodium dodecyl sulfate (SDS) and $30 \%$ glycerol supplemented with $1 \%(\mathrm{w} / \mathrm{v})$ DTT for 15 minutes at room temperature, followed by protein alkylation (carbamidomethylation) in the same equilibration buffer containing $2.5 \%(\mathrm{w} / \mathrm{v})$ iodoacetamide instead of DTT for 15 minutes at room temperature. IPG strips were then placed on the top of $10 \%$ uniform polyacrylamide gels. Strips were overlaid with $0.5 \%$ agarose in $1 \mathrm{x}$ running buffer containing bromophenol blue, and the proteins were further separated by SDS-polyacrylamide gel electrophoresis ( $10 \mathrm{~W}$ per gel) at $20^{\circ} \mathrm{C}$ in the Ettan DALTsix Electrophoresis System (GE Healthcare). After electrophoresis, the gels with CyDyelabeled proteins were directly imaged using a Typhoon ${ }^{\mathrm{TM}}$ Trio image scanner (GE Healthcare, Buckinghamshire, UK).

After 2D-DIGE, gels were scanned on the Typhoon ${ }^{\mathrm{TM}}$ Trio image scanner at different excitation wavelengths (Cy3, 580 BP 30/green [532 nm]; Cy5, 670 BP 30/red [633 nm]; Cy2, 520 BP 40/blue [488 nm]). The intensity was adjusted to ensure that the maximum volume of each image was within 60,000-80,000 U. Analysis of 2D-DIGE was performed using DeCyder 6.5 software (GE Healthcare), according to the manufacturer's recommendations. Briefly, the differential in-gel analysis module was used to detect the intra-gel spots and the biological variation analysis module to match different gels using the in-gel standard. The paired $t$-test was used for statistical analysis of the data. Protein spots that were expressed differentially between two experimental conditions ( $\mid$ ratio $\mid \geq 1.5, P \leq 0.05 t$-test) were marked with master gel numbers. Based on DeCyder 6.5, analysis spots of interest were excised from gels using a Shimadzu Xcise automated gel processing platform (Shimadzu Biotech, Kyoto, Japan). Excised spots were prepared as described previously and then stored at $-20^{\circ} \mathrm{C}$ before analysis by $\mathrm{MS} .{ }^{37}$

\section{MS analysis}

The samples were analyzed by nanoscale capillary liquid chromatography-tandem MS (nano LC-MS/MS). Purification and analysis were performed on a $\mathrm{C} 18$ capillary column using a CapLC system (Waters, Milford, MA) coupled to a hybrid quadrupole orthogonal acceleration time-of-flight tandem mass spectrometer (Q-TOF Ultima; Waters). Chromatographic separation was conducted on a reversed-phase capillary column (Atlantis ${ }^{\mathrm{TM}} \mathrm{dC} 18,3 \mu \mathrm{m}$, $75 \mu \mathrm{m} \times 150 \mathrm{~mm}$ Nano Ease ${ }^{\mathrm{TM}}$; Waters) with a 180-200 $\mathrm{nL} /$ minute flow. The gradient profile consisted of a linear 
gradient from $95 \%$ A (water, $0.1 \%$ formic acid) to $60 \%$ $\mathrm{B}$ ( $80 \%$ acetonitrile, $0.1 \%$ formic acid) in 60 minutes followed by a linear gradient by $95 \%$ B in 10 minutes. Mass data acquisitions were piloted by MassLynx 4.0 software (Micromass Ltd, Manchester, UK) using automatic switching between MS and MS/MS modes. The internal parameters of Q-TOF were set as follows. The electrospray capillary voltage was set to $3.2 \mathrm{kV}$, the cone voltage was set to $30 \mathrm{~V}$, and the source temperature was set to $80^{\circ} \mathrm{C}$. The MS survey scan was $\mathrm{m} / \mathrm{z} 400-1,300$ with a scan time of 1 second and an interscan time of 0.1 second. When the intensity of a peak rose above a threshold of 15 counts, tandem mass spectra were acquired. Normalized collision energies for peptide fragmentation were set using the charge-state recognition files for +2 and +3 peptide ions. The scan range for $\mathrm{MS} / \mathrm{MS}$ acquisition was from $\mathrm{m} / \mathrm{z} 50$ to 1500 with a scan time of 1 second and an interscan time of 0.1 second. Fragmentation was performed using argon as the collision gas and with the collision energy profile optimized for various mass ranges and charges of precursor ions. Mass data collected during a nano LC-MS/MS analysis were processed using ProteinLynx Global Server 2.2 software (Waters) with the following parameters: no background subtraction, smooth 3/2 Savitzky Golay, and no deisotoping to generate peak lists in the Micromass pkl format. Pkl files were then fed into a local search engine Mascot Daemon v2.2.2 (Matrix Science, London, UK). The data were searched against the Homo sapiens (218,356 sequences) and $P$. falciparum (13,110 sequences) National Center for Biotechnology Information nonredundant (NCBInr) protein databases (March 15, 2010). Search parameters allowed for one missed tryptic cleavage site, the carbamidomethylation of cysteine, and the possible oxidation of methionine; precursor and product ion mass error tolerance was $<0.2 \mathrm{Da}$. All identified proteins had a Mascot score greater than 34 and 43 for $P$. falciparum and $H$. sapiens, respectively, corresponding to statistically significant identification $(P<0.05)$.

\section{Results}

\section{SHAM and hyperoxia combinatory effect} on the asexual blood cycle of $P$. falciparum

To study the deleterious effect of SHAM on asexual blood stages of $P$. falciparum in hyperoxia, $21 \%$ oxygen-adapted cultures of $3 \mathrm{D} 7$ were exposed to $\mathrm{SHAM} \mathrm{IC} \mathrm{I}_{50}$. The parasitemia and percentages of the different stages of parasites were evaluated by blood smears at $0,24,32,48$, and 78 hours ( 0 hours corresponding to SHAM addition at the ring stage). In in vitro culture under hyperoxic conditions, the 3D7 P. falciparum strain had a life cycle of 49 hours against 45 hours in normal conditions (Figure 1B). Parasite exposure to $21 \%$ oxygen and SHAM pressure resulted in morphologic alterations involving cellular degeneration during the first 24 hours (Figures 1B and $1 \mathrm{C})$. These results suggest that SHAM addition to 3D7 $P$. falciparum strain under oxidative stress had lethal effects from the beginning of the parasitic cycle and that the life cycle does not continue beyond the ring stage. A previous study demonstrated that hyperoxia induces a delay of $P$. falciparum cycle of 4 hours with an energetic metabolism adaptation crucial to parasite survival. ${ }^{11}$ Consequently, the SHAM/ hyperoxia combinatory effect appeared to result in cellular death, suggesting that the adaptive pathway for hyperoxia was disturbed under SHAM pressure.

\section{Effect of SHAM on P. falciparum asexual blood cycle}

To establish the experimental design for the proteome study, the SHAM $\mathrm{IC}_{50}$ and the action timing of the SHAM should be determined.

Consistent with previously published results, ${ }^{21,38}$ SHAM inhibited growth of the $3 \mathrm{D} 7$ strain with an average $\mathrm{IC}_{50}$ of $246+/-4 \mu \mathrm{M}$.

To determine the SHAM effect on asexual blood stages of P. falciparum, the drug was added at the $\mathrm{IC}_{50}$ concentration on synchronous cultures of 3D7 at the ring stage. The parasitemia and parasitic stage percentages were monitored during two life cycles by blood smears at $0,24,32,48$, and 78 hours in three independent experiments ( 0 hours corresponding to SHAM addition at the ring stage). The parasitemia of SHAMtreated cultures was low at 32 hours compared to untreated parasites (Figures 1A and 1D, 0.6\% parasitemia vs $0.25 \%$, respectively). At the end of 48 hours under SHAM exposure, $54 \%$ of the parasites were degenerated but the parasites not affected by SHAM continued their cycle without phenotypic changes and lengthening of the parasitic cycle. Thus, the SHAM perturbed the parasite growth between the 24th and 32 nd hours of the cycle at $\mathrm{IC}_{50}$ concentration.

\section{SHAM-alteration to the $P$. falciparum proteome}

To study the SHAM effect on $P$. falciparum, a proteomic approach was performed in synchronized parasites exposed to $\mathrm{SHAM} \mathrm{IC}_{50}$. To identify altered $P$. falciparum proteins, 2D-DIGE experiments coupled to MS were performed. Four independent cultures of untreated and SHAM-treated $P$. falciparum were included in this analysis. After protein separation by 2D-DIGE using pH 3-10 IPG strips and 
A

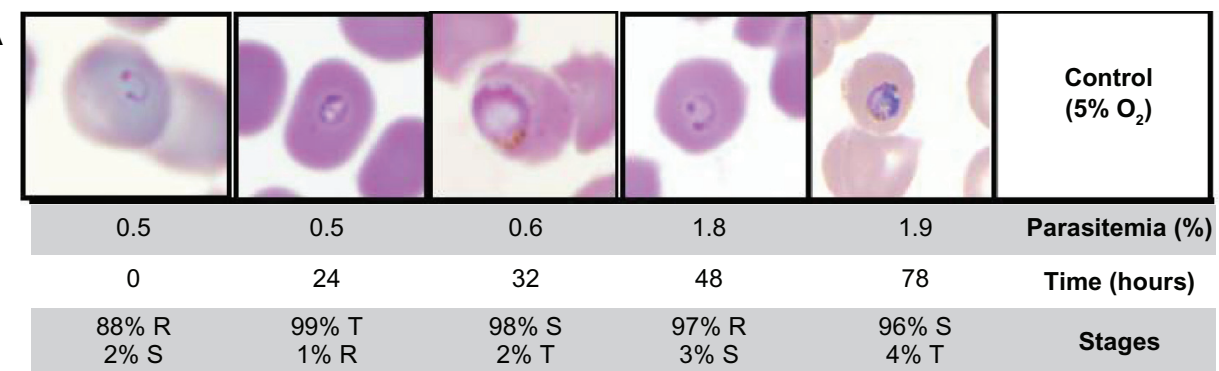

B

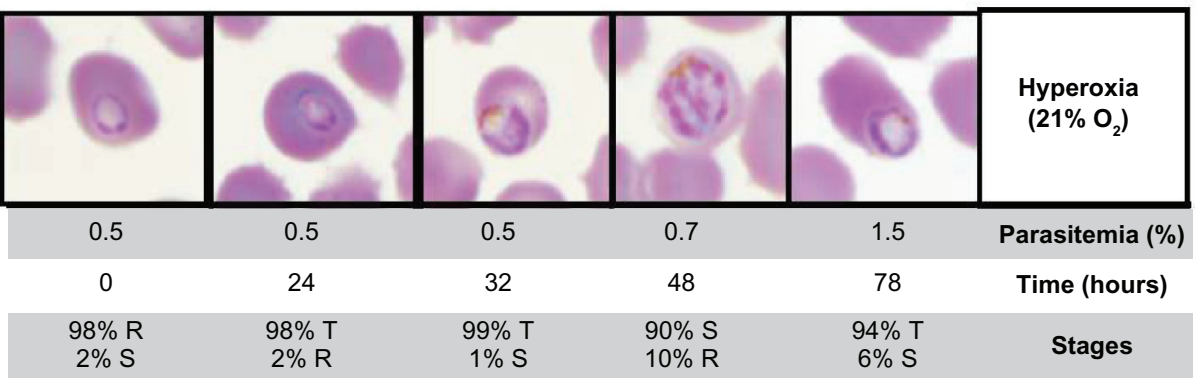

C

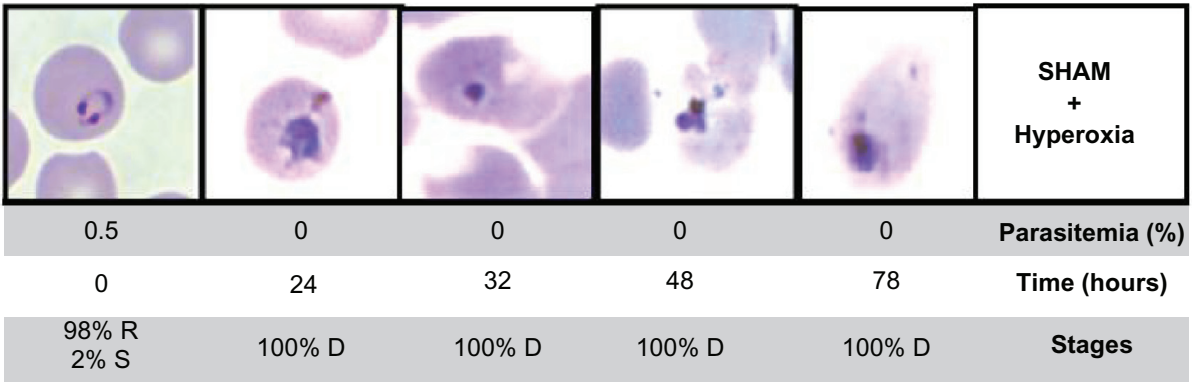

D

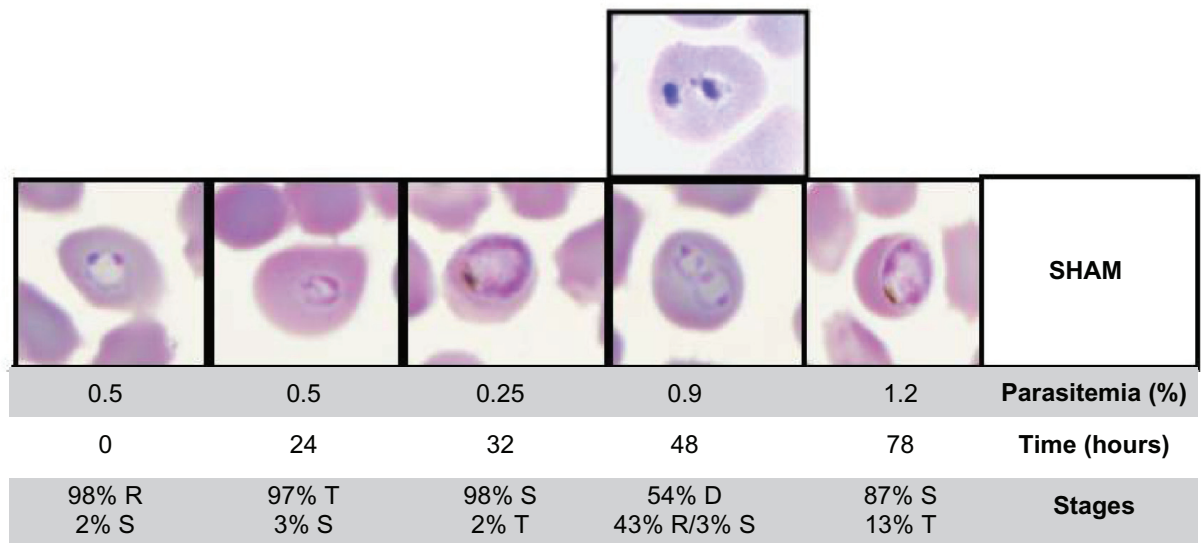

Figure I Morphologic alterations of Plasmodium falciparum following salicylhydroxamic acid (SHAM) pressure. Phenotypic changes of $P$. falciparum asexual blood stages were observed during two parasite cycles under (A) normoxia, (B) hyperoxia (ie, 21\% oxygen), (C) SHAM/hyperoxia conditions, and (D) SHAM pressure. The parasitemia and parasitic stages were evaluated by blood smears at $0,24,32,48$, and 78 hours. After SHAM addition following hyperoxic exposure, significant morphological alterations were observed during the two cycles of parasites. The different intraerythrocytic stages and their corresponding percentages are indicated as follows: ring (R), trophozoites ( $T$ ), schizonts (S), and parasite's death (D).

homogeneous 10\% SDS-polyacrylamide gel electrophoresis, each gel was individually imaged, and all gel images were analyzed using DeCyder 6.5 software. Among 1998 matched protein spots, 18 spots were differentially modulated (|fold change $\mid \geq 1.5, P \leq 0.05 t$-test) between the control and SHAM treatment (seven and eleven spots were up- and down-modulated, respectively; Figure 2). The corresponding protein spots were excised from gels, analyzed by LC-MS/MS, and searched against $P$. falciparum and $H$. sapiens databases (NCBInr). Eight protein spots not fulfilling the selection criteria were still selected and so submitted to MS analysis. Their $P$ values were significant or very close to significance with 


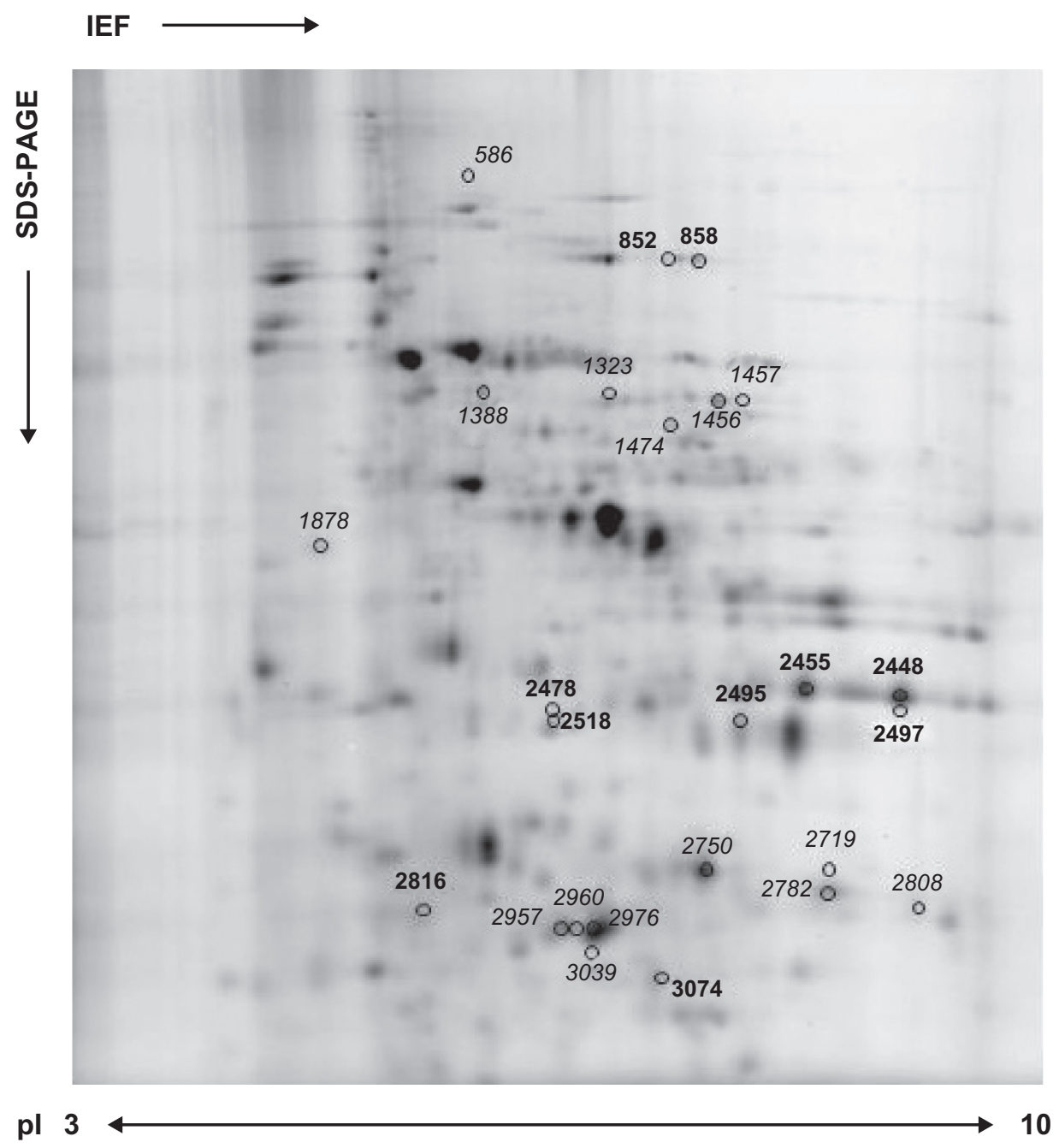

Figure 2 Salicylhydroxamic acid (SHAM) effect on Plasmodium falciparum 3D7 strain proteome. Representative data from a two dimensional-differential gel electrophoresis experiment using a $10 \%$ homogenous sodium dodecyl sulfate polyacrylamide gel with $\mathrm{pH}$ range 3-10 are shown. The proteins from untreated $P$. falciparum parasites or treated parasites with $250 \mu \mathrm{M}$ SHAM were labeled with Cy3 and Cy5, respectively. As determined by DeCyder 6.5 software, protein spots that were up- and down-expressed on $P$. falciparum under SHAM treatment $(|\mathrm{FC}| \geq 1.5, P \leq 0.05 t$-test) were marked with master numbers (Table $I$ ). Bold and italicized numbers correspond, respectively, to identified proteins from $P$. falciparum and Homo sapiens.

$|\mathrm{FC}|$ closed from 1.5 (Table 1). With this last selection, a total of 26 protein spots were identified by MS that correspond to 10 P. falciparum and 16 H. sapiens specific proteins (Table 1). Among these eight last selected proteins, six correspond to isoforms supporting the abundance variation of significant deregulated proteins. Thus, some proteins were detected in more than one spot (eg, four for glyceraldehyde-3-phosphate dehydrogenase, three for catalase), suggesting the presence of different deregulated isoforms. So, the number of distinct proteins identified was five for $P$. falciparum and nine for H. sapiens (Table 1). The importation of human proteins into the parasite during erythrocytic cycle is a well-known phenomenon, as previously described in other studies. ${ }^{39}$ The deregulated protein spots need to be confirmed on other wild P. falciparum strains or isolates.
To determine the metabolic pathways perturbed under SHAM pressure, the identified proteins were classified using the NCBI Clusters of Orthologous Groups of proteins database (Table 1). Among the functional categories, some proteins were predicted to play a role in glycolysis, chaperone-assisted protein folding, and redox metabolism. Among the antioxidant proteins, human catalase, carbonic anhydrase I, and carbonic anhydrase II were altered in SHAM-treated P. falciparum.

\section{Discussion}

\section{SHAM and hyperoxia exposure affects P. falciparum growth}

Previous studies provided evidence for the existence of a P. falciparum $\mathrm{AOX}^{40}$ and supported the ability of SHAM 
Table I Proteins identified from the two dimensional-differential gel electrophoresis analysis in salicylhydroxamic acid-treated Plasmodium falciparum

\begin{tabular}{|c|c|c|c|c|c|c|c|c|}
\hline gi number & $\begin{array}{l}\text { Gene } \\
\text { identification }\end{array}$ & Protein name & MW (kDa) & pl & $\begin{array}{l}\text { Master spot } \\
\text { number }\end{array}$ & $\begin{array}{l}\text { Significance } \\
\text { (Mascot score) }\end{array}$ & $\begin{array}{l}\text { Average } \\
\text { ratio }\end{array}$ & $t$-test \\
\hline \multicolumn{9}{|c|}{ Plasmodium falciparum } \\
\hline \multicolumn{9}{|l|}{ Glycolysis } \\
\hline \multirow[t]{4}{*}{ gi| $\mid 2481013$ | } & PFI4_0598 & $\begin{array}{l}\text { glyceraldehyde-3- } \\
\text { phosphate dehydrogenase }\end{array}$ & 37.06 & 7.59 & $2448^{*}$ & 155 & 1.58 & 0.070 \\
\hline & & & & & 2455 & 67 & 1.64 & 0.024 \\
\hline & & & & & $2495^{*}$ & 47 & 1.66 & 0.057 \\
\hline & & & & & $2497 *$ & 178 & 1.36 & 0.019 \\
\hline \multirow[t]{2}{*}{ gi| 1245 I 3266} & PFI3_0I4I & L-lactate dehydrogenase & 34.33 & 7.12 & $2478^{*}$ & 146 & 1.96 & 0.056 \\
\hline & & & & & 2518 & 175 & 1.72 & 0.049 \\
\hline gi|| 24802328 & PFI0_0I55 & enolase & 49.02 & 6.21 & 2816 & 57 & 1.86 & 0.028 \\
\hline gi|| 24804024 & PFII_0208 & phosphoglycerate mutase & 29.89 & 8.31 & 3074 & 48 & -2.55 & 0.013 \\
\hline \multicolumn{9}{|l|}{ Translation } \\
\hline \multirow[t]{2}{*}{ gi|| 248097 I 2} & PFI4_0486 & elongation factor 2 & 94.54 & 6.36 & 852 & 346 & 1.50 & 0.039 \\
\hline & & & 94.54 & 6.36 & $858 *$ & 279 & 1.42 & 0.051 \\
\hline \multicolumn{9}{|c|}{ Homo sapiens } \\
\hline \multicolumn{9}{|c|}{ Redox metabolism } \\
\hline \multirow[t]{3}{*}{ gi|45025I7 } & & carbonic anhydrase I & 28.91 & 6.59 & 2957 & 136 & -2.26 & 0.013 \\
\hline & & & & & 2960 & 160 & -1.63 & 0.037 \\
\hline & & & & & 2976 & 241 & -3.72 & 0.031 \\
\hline gi|4557395 & & carbonic anhydrase II & 29.28 & 6.87 & 3039 & 135 & -2.26 & 0.029 \\
\hline \multicolumn{9}{|c|}{ Chaperone assisted protein folding } \\
\hline \multicolumn{2}{|c|}{ gi|| $|4| 24984$} & $\begin{array}{l}\text { chaperonin containing TCPI, } \\
\text { subunit } 3 \text { (gamma) }\end{array}$ & 60.93 & 6.10 & 1323 & 96 & 1.49 & 0.0043 \\
\hline \multicolumn{2}{|l|}{ gi|48762932 } & $\begin{array}{l}\text { chaperonin containing TCPI, } \\
\text { subunit } 8 \text { (theta) }\end{array}$ & 60.15 & 5.42 & $1388 *$ & 218 & 1.46 & 0.0067 \\
\hline \multicolumn{9}{|c|}{ Hemoglobin digestion } \\
\hline \multirow[t]{3}{*}{ gi|45570|4 } & & catalase & 59.94 & 6.90 & 1456 & 191 & -3.75 & 0.033 \\
\hline & & & & & 1457 & 219 & -3.63 & 0.029 \\
\hline & & & & & $1474^{*}$ & 185 & -3.29 & 0.055 \\
\hline \multirow[t]{4}{*}{ gi| | 89054 I 78} & & beta-globin & 66.15 & 7.62 & 2719 & 267 & -2.68 & 0.0032 \\
\hline & & & & & 2750 & 244 & -2.15 & 0.0071 \\
\hline & & & & & 2782 & 154 & -4.32 & 0.0067 \\
\hline & & & & & 2808 & 112 & -3.93 & 0.0021 \\
\hline \multicolumn{9}{|c|}{ Pentose phosphate cycle } \\
\hline gi|5803 I87 & & transaldolase I & 37.68 & 6.36 & $2478 *$ & 83 & 1.96 & 0.056 \\
\hline \multicolumn{9}{|c|}{ DNA replication } \\
\hline gi|| 5029922 & & $\mathrm{RNHI}$ protein & 50.10 & 4.83 & 1878 & 114 & 1.58 & 0.024 \\
\hline \multicolumn{9}{|c|}{ Integral membrane proteins } \\
\hline gi|620884I0 & & $\begin{array}{l}\text { spectrin, beta, erythrocytic } \\
\text { variant }\end{array}$ & 269.04 & 5.23 & 586 & 532 & 1.52 & 0.010 \\
\hline
\end{tabular}

Notes: The spot number corresponds to the same numbers as indicated in Figure 2. The Mascot gi number of the spots, their gene identification (gene corresponding as found in PlasmoDB), their name, the theoretical MW, and pl values, as well as the corresponding Mascot score are listed for MS/MS analysis (scores greater than 34 for Plasmodium falciparum and 43 for Homo sapiens are considered as significant $[P<0.05]$ ). Paired average volume ratio (experiment salicylhydroxamic acid versus control) and $P$ values ( $t$-test) were obtained using DeCyder 6.5 software. *Protein spots do not fulfill both criteria of selection, but which were nevertheless submitted to MS analysis thanks to their $P$ values or $F C$ significant.

Abbreviations: MW, molecular weight; pl, isoelectric point, MS, mass spectrometry.

to potentiate the activity of atovaquone, a complex III inhibitor, in in vitro chemosusceptibility assays. ${ }^{21}$ Other studies have examined the effects of cyanide, a complex IV inhibitor, on P. falciparum respiratory metabolism using a polarographic assay. ${ }^{38}$ In the presence of high concentrations of cyanide, $25 \%$ of the parasite oxygen consumption was residual, suggesting that the parasite exploits a cyanide-resistant alternative respiratory pathway. This residual oxygen consumption was totally inhibited by SHAM (coenzyme Q analog), a specific inhibitor of AOX enzymatic activity involved in this alternative pathway. $^{13}$ 
Our results indicate that SHAM $\left(\mathrm{IC}_{50} 250 \mu \mathrm{M}\right)$ induced the death of effectively $54 \%$ of the parasites but in hyperoxic conditions and to the same $\mathrm{IC}_{50}$, parasite death is $100 \%$. Therefore, in hyperoxic conditions, SHAM exposure has a deleterious effect on parasite survival. SHAM seems to inhibit the metabolic adaptation involved in hyperoxia, which is essential to parasite survival.

Consequently, as described in plants, ${ }^{23,24}$ SHAM action seems to prevent the decrease in the levels of reactive oxygen species (ROS), which are exceedingly produced in hyperoxia by the mETC. ${ }^{42}$ Because the AOX activity reduces oxygen, its activity should be dependent on the oxygen concentration and also on the coenzyme $\mathrm{Q}$ concentration and its redox state. ${ }^{23}$ This alternative pathway could provide a regulatory function for excess electrons when the mETC is saturated (as during hyperoxia) and thus, decrease ATP production (unpublished data). Additionally, this "electron leak" mechanism may improve potential repression of the TCA cycle by elevated levels of NADH and ATP. ${ }^{24}$

\section{SHAM alters the $P$. falciparum proteome}

To investigate the metabolic pathways altered by the activity of SHAM, a comparative study on the P. falciparum proteome was performed using 2D-DIGE coupled to MS for identification. The experimental design (parasite stage, drug dose, incubation time) was chosen based on our preliminary results. In our study, SHAM was exposed at the $\mathrm{IC}_{50}$ concentration to ring stage parasites for 12 hours.

Most SHAM-altered proteins are involved in glycolysis and redox metabolism. The major implication of glycolytic enzymes (glyceraldehyde-3-phosphate dehydrogenase, L-lactate dehydrogenase, enolase, and phosphoglycerate mutase) suggests the need to sustain the major energy dependence of the parasite on glycolysis. Although the function of the $P$. falciparum mitochondrion remains unclear, its contribution to ATP production is considered to be minimal in asexual stages. P. falciparum relies principally on anaerobic glycolysis for energy production. ${ }^{4}$ Therefore, the parasites are dependent on glycolysis in a microaerophilic environment while in hyperoxia conditions; our previous results showed that the respiratory metabolism is favored at the expense of glycolysis. ${ }^{11}$ This observation could explain the lethal effect of SHAM in hyperoxic conditions. The NADH generated during glycolysis are reoxidized through glycerol-3-phosphate in the cytosol and by PfNDH2 in the mitochondria. ${ }^{43,44}$ In plants, the alternative respiratory pathway involves an alternative NADH dehydrogenase, coenzyme Q, and AOX..$^{23}$ Mitochondrial AOX exists under two forms, covalent and noncovalent homodimers. ${ }^{45}$ The oxidized covalent form of the enzyme is inactive, whereas reducing the disulfide bond generated by NADH activates the enzyme, possibly mediated by a glutathione/thioredoxin coupling system. ${ }^{46}$ Interestingly, glycolysis activation under SHAM pressure could be the result of a metabolic adaptation in a microaerophilic environment. The end products of $P$. falciparum glycolysis are pyruvate, lactate, and glycerol. Firstly, pyruvate is an allosteric activator of AOX counterbalancing the AOX inhibition by SHAM. Under conditions of partial respiration, accumulation of pyruvate would result in increased NADH, which could potentially activate AOX. ${ }^{2}$ Secondly, lactate, via L-lactate dehydrogenase, may be a substrate for the mETC under stress conditions. ${ }^{43}$ Finally, glycerol production by the glycerol-3-phosphate permitted the reoxidation of NADH and the activity of the mETC ${ }^{47}$ (Figure 3). In a microaerophilic environment, the activation of glycolysis is concordant with the role of glycolytic metabolism in the reoxidation of $\mathrm{NADH},{ }^{48}$ an essential cofactor of the alternative respiratory pathway in T. bruce $i^{26}$ and C. parvum. ${ }^{44}$ Indeed, T. brucei brucei survives using an anaerobic pathway while T. brucei brucei AOX is inhibited. ${ }^{26,41}$ Collectively, these results showed that SHAM, via its action on glycolysis metabolism, appears to play a role in NAD/NADH balance. The mechanism of regulation

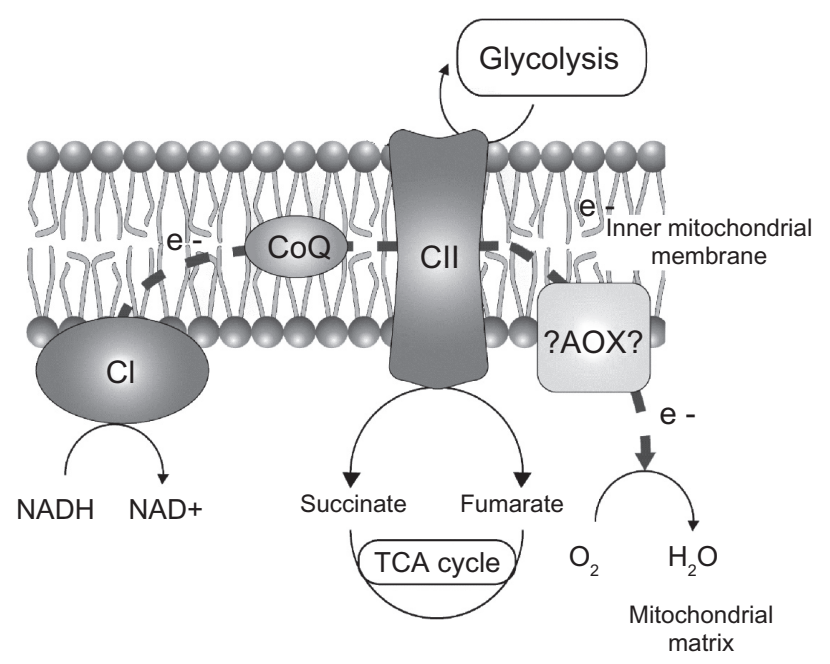

Figure 3 A schematic representation of the Plasmodium falciparum metabolic pathways perturbed under salicylhydroxamic acid treatment. Glycolysis and mitochondrial respiratory chain pathways based on the Ginsburg website are represented. ${ }^{50}$ 
of AOX activity involved several compounds, including those in redox states (NAD/NADH) and allosteric effectors (pyruvate). ${ }^{49}$ Thus, the existence of an AOX function in $P$. falciparum could be beneficial for the survival of the parasite in hyperoxic environments as in the salivary glands of mosquitoes or perhaps in the human lungs.

SHAM treatment leads to an inhibition of antioxidant proteins. Among the proteins identified, some originated from the human erythrocyte host and correspond to host proteins imported by the parasite or proteins arising from host-parasite interaction. Consequently, cytosolic catalase and carbonic anhydrase I were down-represented under SHAM pressure. While $P$. falciparum lacks the antioxidant enzyme catalase, host catalase was imported into the food vacuole ${ }^{39,50}$ and accomplished hydrogen peroxide detoxification. ${ }^{51}$ Thus, the down-importation of catalase could be attributed to a SHAM effect against oxidative damage by diminishing hydrogen peroxide production. Carbonic anhydrase has been identified as a major intracellular peroxidation target in erythrocytes and could be attributed to oxidative stress induced by SHAM in the parasitized erythrocytes. ${ }^{52}$

In our study, a number of identified proteins (enolase, catalase, glyceraldehyde-3-phosphate dehydrogenase, carbonic anhydrase) have been previously found as potential glutaredoxin target proteins. ${ }^{53}$ In higher plants, the reduction of AOX in its active form involved a glutathione/ thioredoxin coupling system, suggesting a SHAM effect on feedback regulation of AOX activity as described in higher plants. ${ }^{24,46}$

The other deregulated proteins were particularly involved in protein processing (chaperonin-containing T-complex 1), as well as translation (elongation factor 2), consequences of a general stress state of the parasite. Chaperone-assisted protein folding was described to contribute to protein folding, assembly, and translocation, inducing expression of proteins in these pathways under several types of cellular stress. $^{54}$

\section{Conclusion}

Although the AOX gene was not characterized yet in the P. falciparum, SHAM, an AOX specific inhibitor, disturbed the in vitro growth of $P$. falciparum in hyperoxic conditions. Additionally, recent studies sustained the existence of the alternative respiratory pathway in P. falciparum, illustrated by the identification of alternative NADH dehydrogenase, mostly described in some detail for plants. ${ }^{13,55}$ Despite the fact that the P. falciparum AOX gene was not yet identified, the metabolic pathways involved in SHAM response illustrates the existence of a similar AOX function. The failure of a comparative sequence analysis is mainly due to the extreme adenine-thymine bias (80\%) of the parasite genome or the high gene variability between other species and $P$. falcipar$u m .{ }^{56}$ Nevertheless, an AOX-like function could play a role in oxidative stress defense and could be a major benefit to P. falciparum, which appears to be sensitive to hyperoxic conditions. Indeed, the respiratory metabolism was the predominant source of ROS on $P$. falciparum, and partial inhibition of this metabolism led to a decrease of ROS. This hypothesis is in agreement with a publication reporting the AOX protective role in preventing ROS production. ${ }^{23}$

Several studies on the use of mETC inhibitors underlined the crucial role of respiratory metabolism to parasite survival. ${ }^{9}$ However, much work remains to scrutinize the specific biochemical pathways of the $P$. falciparum mitochondria. ${ }^{8}$ This unique particularity of the alternative respiratory pathway could be a promising target for the development of a new group of antimalarial drugs.

\section{Acknowledgments}

The authors acknowledge the financial support of Délégation Générale pour l'Armement and the Direction Centrale du Service de Santé des Armées (grant no. 06co008). This paper has been reviewed and corrected by a journal expert.

\section{Disclosure}

The authors report no conflicts of interest in this work.

\section{References}

1. Fry M, Beesley JE. Mitochondria of mammalian Plasmodium spp. Parasitology. 1991;102 Pt 1:17-26.

2. Mi-Ichi F, Takeo S, Takashima E, et al. Unique properties of respiratory chain in Plasmodium falciparum mitochondria. Adv Exp Med Biol. 2003; 531:117-133

3. Ralph SA. Strange organelles-Plasmodium mitochondria lack a pyruvate dehydrogenase complex. Mol Microbiol. 2005;55(1):1-4.

4. Van Dooren GG, Stimmler LM, McFadden GI. Metabolic maps and functions of the Plasmodium mitochondrion. FEMS Microbiol Rev. 2006;30(4):596-630.

5. Fry M, Webb E, Pudney M. Effect of mitochondrial inhibitors on adenosinetriphosphate levels in Plasmodium falciparum. Comp Biochem Physiol B. 1990;96(4):775-782.

6. Painter HJ, Morrisey JM, Mather MW, Vaidya AB. Specific role of mitochondrial electron transport in blood-stage Plasmodium falciparum. Nature. 2007;446(7131):88-91.

7. Mogi T, Kita K. Identification of mitochondrial complex II subunits SDH3 and SDH4 and ATP synthase subunits a and b in Plasmodium spp. Mitochondrion. 2009;9(6):443-453.

8. Torrentino-Madamet M, Desplans J, Travaillé C, Jammes Y, Parzy D. Microaerophilic respiratory metabolism of Plasmodium falciparum mitochondrion as a drug target. Curr Mol Med. 2010;10(1):29-46. 
9. Vaidya AB. Mitochondrial and plastid functions as antimalarial drug targets. Curr Drug Targets Infect Disord. 2004;4(1):11-23.

10. Mather MW, Henry KW, Vaidya AB. Mitochondrial drug targets in apicomplexan parasites. Curr Drug Targets. 2007;8(1):49-60.

11. Torrentino-Madamet M, Alméras L, Desplans J, et al. Global response of Plasmodium falciparum to hyperoxia: a combined transcriptomic and proteomic approach. Malar J. 2011;10:4.

12. Uyemura SA, Luo S, Vieira M, Moreno SN, Docampo R. Oxidative phosphorylation and rotenone-insensitive malate- and NADH-quinone oxidoreductases in Plasmodium yoelii yoelii mitochondria in situ. $J$ Biol Chem. 2004;279(1):385-393.

13. Biagini GA, Viriyavejakul P, O’Neill PM, Bray PG, Ward SA. Functional characterization and target validation of alternative complex I of Plasmodium falciparum mitochondria. Antimicrob Agents Chemother. 2006;50(5):1841-1851.

14. Dong CK, Patel V, Yang JC, et al. Type II NADH dehydrogenase of the respiratory chain of Plasmodium falciparum and its inhibitors. Bioorg Med Chem Lett. 2009;19(3):972-975.

15. Martin W, Muller M. The hydrogen hypothesis for the first eukaryote. Nature. 1998;392(6671):37-41.

16. Lopez-Garcia P, Moreira D. Metabolic symbiosis at the origin of eukaryotes. Trends Biochem Sci. 1999;24(3):88-93.

17. Van der Giezen M, Tovar J. Degenerate mitochondria. EMBO Rep. 2005;6(6):525-530.

18. Smilkstein MJ, Forquer I, Kanazawa A, et al. A drug-selected Plasmodium falciparum lacking the need for conventional electron transport. Mol Biochem Parasitol. 2008;159(1):64-68.

19. Srivastava IK, Rottenberg H, Vaidya AB. Atovaquone, a broad spectrum antiparasitic drug, collapses mitochondrial membrane potential in a malarial parasite. J Biol Chem. 1997;272(7):3961-3966.

20. Legrand E, Demar M, Volney B, et al. First case of emergence of atovaquone resistance in Plasmodium falciparum during second-line atovaquone-proguanil treatment in South America. Antimicrob Agents Chemother. 2007;51(6):2280-2281.

21. Murphy AD, Lang-Unnasch N. Alternative oxidase inhibitors potentiate the activity of atovaquone against Plasmodium falciparum. Antimicrob Agents Chemother. 1999;43(3):651-654.

22. Schonbaum GR, Bonner WD Jr, Storey BT, Bahr JT. Specific inhibition of the cyanide-insensitive respiratory pathway in plant mitochondria by hydroxamic acids. Plant Physiol. 1971;47(1):124-128.

23. Juszczuk IM, Rychter AM. Alternative oxidase in higher plants. Acta Biochim Pol. 2003;50(4):1257-1271.

24. Vanlerberghe GC, McIntosh L. ALTERNATIVE OXIDASE: from gene to function. Annu Rev Plant Physiol Plant Mol Biol. 1997;48:703-734.

25. Divo AA, Geary TG, Jensen JB, Ginsburg H. The mitochondrion of Plasmodium falciparum visualized by rhodamine 123 fluorescence. J Protozool. 1985;32(3):442-446.

26. Chaudhuri M, Ott RD, Hill GC. Trypanosome alternative oxidase: from molecule to function. Trends Parasitol. 2006;22(10):484-491.

27. Bar Nun N, Plakhine D, Joel DM, Mayer AM. Changes in the activity of the alternative oxidase in Orobanche seeds during conditioning and their possible physiological function. Phytochemistry. 2003;64(1): 235-241.

28. Roberts CW, Roberts F, Henriquez FL, et al. Evidence for mitochondrialderived alternative oxidase in the apicomplexan parasite Cryptosporidium parvum: a potential anti-microbial agent target. Int J Parasitol. 2004;34(3):297-308.

29. Suzuki T, Hashimoto T, Yabu Y, et al. Alternative oxidase (AOX) genes of African trypanosomes: phylogeny and evolution of AOX and plastid terminal oxidase families. J Eukaryot Microbiol. 2005;52(4): 374-381.

30. Suzuki T, Nihei C, Yabu Y, et al. Molecular cloning and characterization of Trypanosoma vivax alternative oxidase (AOX) gene, a target of the trypanocide ascofuranone. Parasitol Int. 2004;53(3):235-245.

31. Briolant S, Almeras L, Belghazi M, et al. Plasmodium falciparum proteome changes in response to doxycycline treatment. Malar J. 2010; $9: 141$.
32. Desjardins RE, Canfield CJ, Haynes JD, Chulay JD. Quantitative assessment of antimalarial activity in vitro by a semiautomated microdilution technique. Antimicrob Agents Chemother. 1979;16(6): 710-718.

33. Trager W, Jensen JB. Human malaria parasites in continuous culture. 1976. J Parasitol. 2005;91(3):484-486.

34. Lambros C, Vanderberg JP. Synchronization of Plasmodium falciparum erythrocytic stages in culture. J Parasitol. 1979;65(3):418-420.

35. Uhlemann AC, Staalsoe T, Klinkert MQ, Hviid L. Analysis of Plasmodium falciparum-infected red blood cells. MACS \& more. 2000; 4(2):7-8.

36. Pastorino B, Boucomont-Chapeaublanc E, Peyrefitte CN, et al. Identification of cellular proteome modifications in response to West Nile virus infection. Mol Cell Proteomics. 2009;8(7):1623-1637.

37. Shevchenko A, Wilm M, Vorm O, Mann M. Mass spectrometric sequencing of proteins silver-stained polyacrylamide gels. Anal Chem. 1996;68(5):850-858.

38. Murphy AD, Doeller JE, Hearn B, Lang-Unnasch N. Plasmodium falciparum: cyanide-resistant oxygen consumption. Exp Parasitol. 1997;87(2):112-120.

39. Koncarevic S, Rohrbach P, Deponte M, et al. The malarial parasite Plasmodium falciparum imports the human protein peroxiredoxin 2 for peroxide detoxification. Proc Natl Acad Sci U S A. 2009;106(32): 13323-13328.

40. Krungkrai J, Burat D, Kudan S, Krungkrai S, Prapunwattana P. Mitochondrial oxygen consumption in asexual and sexual blood stages of the human malarial parasite, Plasmodium falciparum. Southeast Asian J Trop Med Public Health. 1999;30(4):636-642.

41. Helfert S, Estevez AM, Bakker B, Michels P, Clayton C. Roles of triosephosphate isomerase and aerobic metabolism in Trypanosoma brucei. Biochem J. 2001;357(Pt 1):117-125.

42. Oliveira PL, Oliveira MF. Vampires, Pasteur and reactive oxygen species. Is the switch from aerobic to anaerobic metabolism a preventive antioxidant defence in blood-feeding parasites? FEBS Lett. 2002; 525(1-3):3-6.

43. Rasmusson AG, Geisler DA, Moller IM. The multiplicity of dehydrogenases in the electron transport chain of plant mitochondria. Mitochondrion. 2008;8(1):47-60.

44. Henriquez FL, Richards TA, Roberts F, McLeod R, Roberts CW. The unusual mitochondrial compartment of Cryptosporidium parvum. Trends Parasitol. 2005;21(2):68-74.

45. Rhoads DM, Umbach AL, Sweet CR, Lennon AM, Rauch GS, Siedow JN. Regulation of the cyanide-resistant alternative oxidase of plant mitochondria. Identification of the cysteine residue involved in alphaketo acid stimulation and intersubunit disulfide bond formation. $J$ Biol Chem. 1998;273(46):30750-30756.

46. Arnholdt-Schmitt B, Costa JH, de Melo DF. AOX - a functional marker for efficient cell reprogramming under stress? Trends Plant Sci. 2006; 11(6):281-287.

47. Lian LY, Al-Helal M, Roslaini AM, et al. Glycerol: an unexpected major metabolite of energy metabolism by the human malaria parasite. Malar J. 2009;8:38.

48. Vaidya AB, Mather MW. Mitochondrial evolution and functions in malaria parasites. Annu Rev Microbiol. 2009;63:249-267.

49. Sluse FE, Jarmuszkiewicz W. Alternative oxidase in the branched mitochondrial respiratory network: an overview on structure, function, regulation, and role. Braz J Med Biol Res. 1998;31(6):733-747.

50. Ginsburg, Hagai. Malaria parasite metabolic pathways. January 11, 2000. Available from: http://sites.huji.ac.il/malaria. Accessed July 28, 2011.

51. Clarebout G, Slomianny C, Delcourt P, et al. Status of Plasmodium falciparum towards catalase. Br J Haematol. 1998;103(1):52-59.

52. Wiwanitkit V. Plasmodium and host carbonic anhydrase: molecular function and biological process. Gene Ther Mol Biol. 2006;10(2): $251-254$.

53. Sturm N, Jortzik E, Mailu BM, et al. Identification of proteins targeted by the thioredoxin superfamily in Plasmodium falciparum. PLoS Pathog. 2009;5(4):e1000383. 
54. Acharya P, Kumar R, Tatu U. Chaperoning a cellular upheaval in malaria: heat shock proteins in Plasmodium falciparum. Mol Biochem Parasitol. 2007; 153(2):85-94.

55. Fisher N, Bray PG, Ward SA, Biagini GA. The malaria parasite type II NADH: quinone oxidoreductase: an alternative enzyme for an alternative lifestyle. Trends Parasitol. 2007;23(7):305-310.
56. Paila U, Kondam R, Ranjan A. Genome bias influences amino acid choices: analysis of amino acid substitution and re-compilation of substitution matrices exclusive to an AT-biased genome. Nucleic Acids Res. 2008;36(21):6664-6675.

\section{Publish your work in this journal}

Research and Reports in Tropical Medicine is an international, peerreviewed, open access journal publishing original research, case reports, editorials, reviews and commentaries on all areas of tropical medicine, including: Diseases and medicine in tropical regions; Entomology; Epidemiology; Health economics issues; Infectious disease; Laboratory science and new technology in tropical medicine; Parasitology; Public health medicine/health care policy in tropical regions; and Microbiology. The manuscript management system is completely online and includes a very quick and fair peer-review system. Visit http://www.dovepress. com/testimonials.php to read real quotes from published authors.

Submit your manuscript here: http://www.dovepress.com/research-and-reports-in-tropical-medicine-journal 